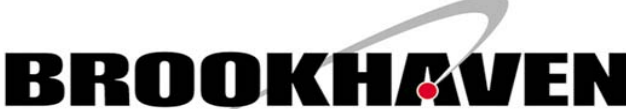 \\ NATIONAL LABORATORY
}

BNL-113658-2017-CP

\section{Fluctuations in neutron-induced fission cross sections}

\author{
G.F. Bertsch, David Brown \\ Submitted to the Sixth International Conference on \\ Fission and Properties of Neutron-rich Nuclei \\ Held at Sundial Beach Resort, Sanibel Island, Florida \\ November 6-12, 2016
}

November 2017

\author{
National Nuclear Data Center \\ Brookhaven National Laboratory
}

\section{U.S. Department of Energy Office of Science, Office of Nuclear Physics}

\footnotetext{
Notice: This manuscript has been authored by employees of Brookhaven Science Associates, LLC under Contract No.DE-SC0012704 with the U.S. Department of Energy. The publisher by accepting the manuscript for publication acknowledges that the United States Government retains a non-exclusive, paid-up, irrevocable, world-wide license to publish or reproduce the published form of this manuscript, or allow others to do so, for United States Government purposes.
} 


\section{DISCLAIMER}

This report was prepared as an account of work sponsored by an agency of the United States Government. Neither the United States Government nor any agency thereof, nor any of their employees, nor any of their contractors, subcontractors, or their employees, makes any warranty, express or implied, or assumes any legal liability or responsibility for the accuracy, completeness, or any third party's use or the results of such use of any information, apparatus, product, or process disclosed, or represents that its use would not infringe privately owned rights. Reference herein to any specific commercial product, process, or service by trade name, trademark, manufacturer, or otherwise, does not necessarily constitute or imply its endorsement, recommendation, or favoring by the United States Government or any agency thereof or its contractors or subcontractors. The views and opinions of authors expressed herein do not necessarily state or reflect those of the United States Government or any agency thereof. 


\title{
Fluctuations in neutron-induced fission cross sections
}

\author{
G.F. Bertsch ${ }^{1}$ \\ Department of Physics and Institute of Nuclear Theory, Box 351560 \\ University of Washington, Seattle, Washington 98915, USA \\ David Brown \\ National Nuclear Data Center, Brookhaven National Laboratory, Upton \\ $N Y, U S A$
}

\begin{abstract}
We examine the published experimental data on the ${ }^{235} \mathrm{U}(\mathrm{n}, \mathrm{f})$ reaction with a goal to quantify cross section fluctuations above the fission barrier. For neutron energies in the range $10 \mathrm{keV}-25 \mathrm{keV}$ we find that there are significant fluctuations of the order of $5 \%$ measured at 0.25 $\mathrm{keV}$ energy resolution. Here we analyze the fluctuations by the autocorrelation function with the following conclusions: 1) The correlation width associated with peak at zero energy offset is smaller than the experimental energy resolution; 2) there are peaks in the autocorrelation function on an energy scale of several keV. Such a multi-peaked structure is incompatible with Ericson's statistical treatment of compound nucleus fluctuations but may arise in more detailed models of the fission dynamics.
\end{abstract}

\section{Introduction}

The dynamics of fission above the fission barrier is far from well understood. Present theory [1] is largely based on the Bohr-Wheeler framework [2] which introduced the concept of fission channels. The channels are characterized by their energy-dependent transmission coefficients $T(E)$ but there still no predictive theory to calculate them. Commonly $T$ is parameterized by the Hill-Wheeler formula [3, p. 1140],

$$
T(E)=\left(1+\exp \left(2 \pi\left(E-E_{B}\right) / \hbar \omega\right)\right)^{-1}
$$

where $E_{B}$ is the barrier height and the $\omega$ is a frequency associated with the thickness of the barrier. However, there is no compelling experimental data to support the Hill-Wheeler parameterization. The transmission coefficients could equally well be described as resonant states at the barrier top, in analogy to quantum dots bridging conductors in the mesoscopic theory of electron transport [8]. The corresponding formula is

$$
T(E)=\Gamma_{R} \Gamma_{L} /\left(\left(E-E_{r}\right)^{2}+\left(\Gamma_{R}+\Gamma_{L}\right)^{2} / 4\right) .
$$

\footnotetext{
${ }^{1}$ bertsch@uw.edu
} 
This depends on three parameters, namely the resonance energy and the decay widths to the left and right. Measurement of the fluctuations in the cross section may provide a way to distinguish between these possibilities. In the Hill-Wheeler approach, the channels themselves introduce very little fluctuations-they open over an energy range of the order of $\hbar \omega \sim 1 \mathrm{MeV}$, and once open they remain so for all higher energy. The only mechanism to introduce fluctuations on a finer scale is Ericson's theory [4] treating the statistical feeding of the channels from inside the defining barrier. In contrast, the picture of the channels as resonant states bridging the barrier permits arbitrary fluctuations associated with the widths $\Gamma_{R}$ and $\Gamma_{L}$.

With this mind, we examine here the experimental evidence for fluctuations above the fission barrier. There is much data on neutron-induced reaction on ${ }^{235} \mathrm{U}$ including the energy dependence of fission, capture, and total cross sections. The fluctuations associated with different entrance channels are likely to be independent, and the best opportunity to observe them is when the number of entrance channels is small. For neutron energies below a few $\mathrm{keV}$, cross sections are dominated by $s$-wave neutrons with two total angular momentum channels for an odd-A target such as ${ }^{235} \mathrm{U}$. With this in mind, we focus on the data for the range of neutron energies from 10 to $25 \mathrm{keV}$.

\section{The data}

Three independent measurements of the reaction ${ }^{235} \mathrm{U}(\mathrm{n}, \mathrm{f})$ in the energy range $E_{n}=10-25 \mathrm{keV}$ are shown in Fig. 1. It is obvious that there is some fluctuation outside of the limits of experimental error. For example, note the prominent peak at $22 \mathrm{keV}$, present in all three measurements. The peak could arise from a doorway entrance channel or a transmission factor peak from in a resonant-state fission channel. The remaining possibility, a localized dip in the capture cross section is very unlikely due to the large number of capture channels.

We start with a very elementary test for fluctuations. This is the variance of the measured data with respect to a polynomial fit. Here we make a quadratic fit

$$
\sigma_{f i t}(E)=\bar{\sigma}+a(E-\bar{E})+b(E-\bar{E})^{2}
$$

to each data set and compare the variance of the fit error

$$
v=\left(\frac{1}{N_{d}} \sum_{i}^{D_{d}}\left(\sigma_{e x p}\left(E_{i}\right)-\sigma_{f i t}\left(E_{i}\right)\right)^{2}\right)^{1 / 2}
$$




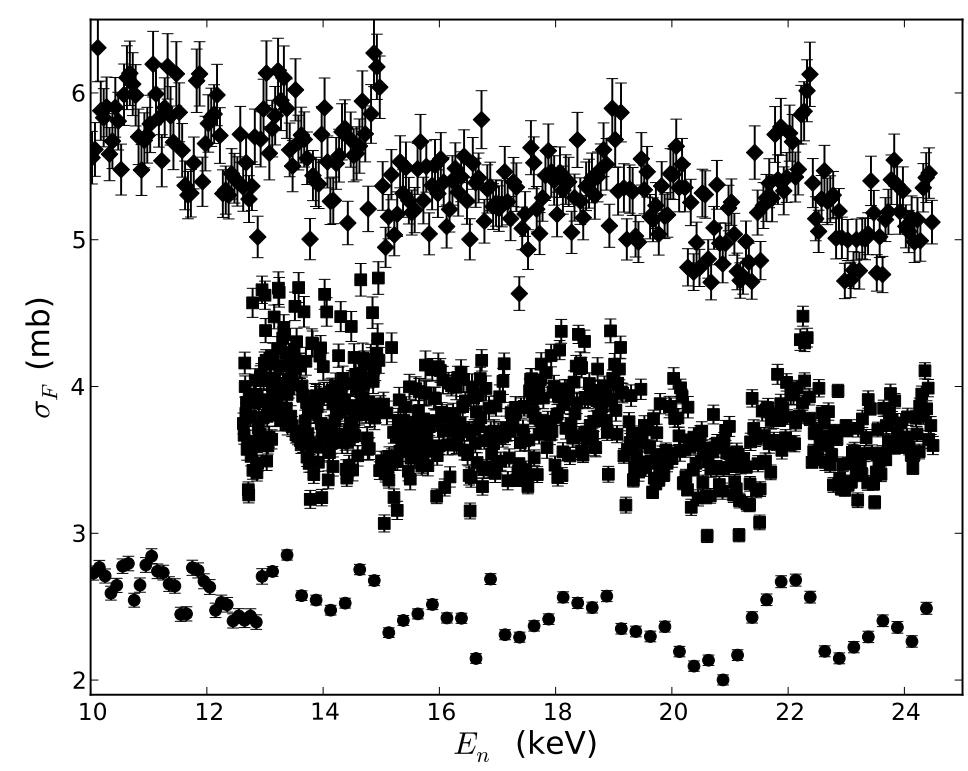

Fig. 1. Experimental fission cross sections in the range $10-25 \mathrm{keV}$. Circles: from Ref. [7]; squares: from [5]; diamonds: from [6]. The latter two data sets have been shifted upward for clarity in the plotting the figure.

to the reported experimental error bar $v_{\text {exp }}$. The results are shown in Table I. The variance for the data on the top line is nearly 4 times larger than the

\begin{tabular}{|cc|ccc|cc|}
\hline $\begin{array}{c}\text { data range } \\
\mathrm{keV}\end{array}$ & source & $\begin{array}{c}\bar{\sigma} \\
\mathrm{b}\end{array}$ & $\begin{array}{c}a \\
\mathrm{~b} / \mathrm{keV}\end{array}$ & $\begin{array}{c}b \\
\mathrm{~b} / \mathrm{keV}^{2}\end{array}$ & $\begin{array}{c}v \\
\mathrm{~b}\end{array}$ & $\begin{array}{c}v_{\exp } \\
\mathrm{b}\end{array}$ \\
\hline $10-24.5$ & {$[7]$} & 2.4521 & -0.0331 & 0.00250 & 0.15 & 0.04 \\
$12.6-24.5$ & {$[5]$} & 2.2844 & -0.0240 & 0.00348 & 0.25 & 0.06 \\
$10-25$ & {$[6]$} & 2.3415 & -0.0457 & 0.00362 & 0.27 & 0.17 \\
\hline
\end{tabular}

average quoted experimental error. The variance for the data on the other lines is even larger. We conclude that fluctuations in the cross sections are present at keV energy scales and can be measured. In conventional theory, the only way to explain them is by Ericson fluctuations associated with the compound nucleus states in either the first or second wells of the fission potential energy surface. 


\section{Autocorrelation analysis}

More detailed information about the fluctuations can be obtained by an analysis of the autocorrelation function. The usual formulation assumes a constant average cross section, but this is not tenable over a large range of energies. We shall apply the analysis by examining the correlation in the reduced cross section $\tilde{\sigma}(E)$ obtained by normalizing to the fit function $\sigma_{f i t}(E)$.

$$
\tilde{\sigma}(E)=\frac{\sigma(E)}{\sigma_{f}(E)} .
$$

The autocorrelation function is then defined as

$$
C(\epsilon)=\langle\tilde{\sigma}(E) \tilde{\sigma}(E+\epsilon)\rangle
$$

As shown by Ericson [4], the correlation function can be simply interpreted if the reaction is mediated by independent compound-nucleus resonances feeding the appropriate exit channels. Then it has the form

$$
C(\epsilon) \approx 1+\frac{1}{N} \frac{1}{1+(\epsilon / \Gamma)^{2}}
$$

where $\Gamma$ is the average decay width of the resonances and $N$ is the effective number of exit channels.

We show in Fig. 2 the results for the data from [7] in the range $10-25$ $\mathrm{keV}$, using the quadratic fit for the average cross section. The experimental data was partially rebinned with a bin size $E_{B}=0.25 \mathrm{keV}$ to calculate $C$ in steps of $E_{B}$. The error bars on the graph were obtained by sampling data sets with random errors added to the experimental values. The added errors are Gaussian distributed with a variance given by the quoted experimental error. We see that the correlation function has a multi-peak structure. The first peak at $\epsilon=0$ is very sharp, having an excess of 0.0043 and falling immediately to 1 in the next bin at $\epsilon=0.25 \mathrm{keV}$. It is clear that $\Gamma$ in the Ericson formula must be much less than the bin size. In that limit, the excess can be interpret by the formula [9]

$$
C(0)-1=\frac{1}{N} \frac{1}{1+\left(E_{B} / \pi \Gamma\right)} .
$$

The average width $\Gamma$ of the compound nucleus states in the first well can be reliably estimated as $\Gamma \approx 0.2 \mathrm{eV}$. Eq. (8) then yields $N \approx 0.6$ which is much too small to be compatible with the experimental reaction cross section [10]. However, the existence of other peaks in $C$ renders the entire Ericson fluctuation analysis problematic. The correlation function does not 


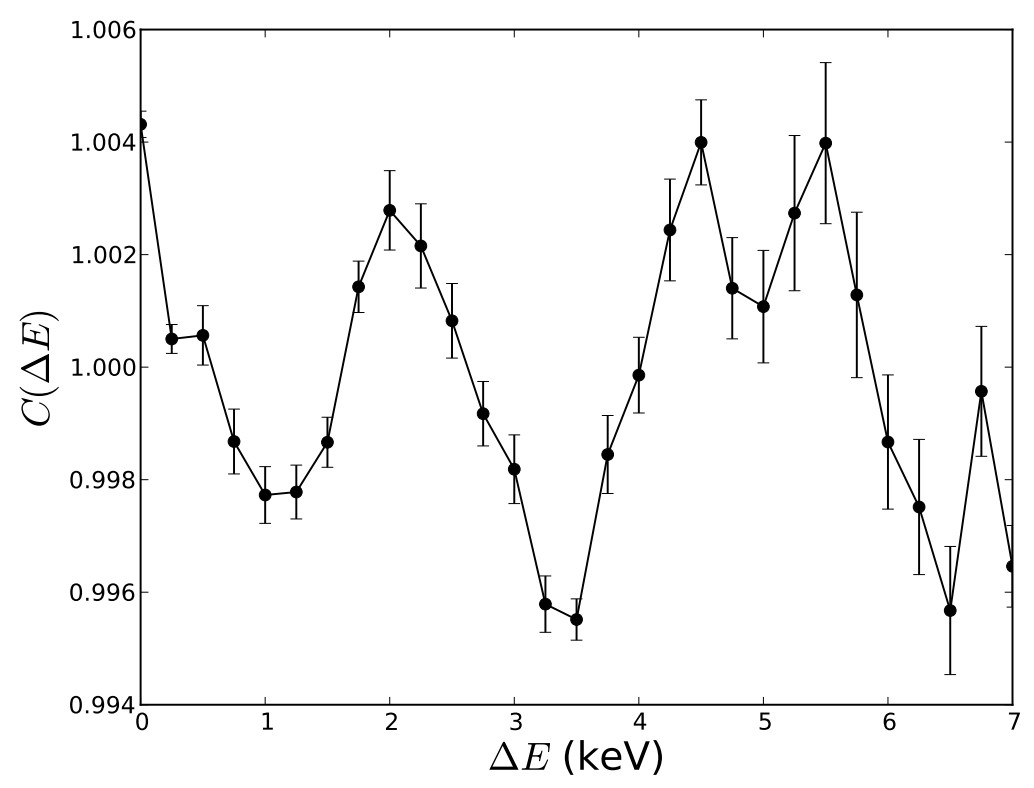

Fig. 2. Autocorrelation function for ${ }^{235} \mathrm{U}(\mathrm{n}, \mathrm{f})$ in the range fission cross sections in the range $10-25 \mathrm{keV}$ for the data in Ref. [7].

look anything like Eq. (7), deviating more from unity at finite $\epsilon$ than at $\epsilon=0$. We have not shown the correlation functions extracted from the other data sets cited in Table I, but they also show a multi-peak structure similar to that exhibited in Fig. 2. Obviously, the dynamics are far more complicated than the compound nucleus picture permits.

\section{Outlook}

There are several possibilities to gain some understanding of the measured autocorrelation function. The first that comes to mind that doorway states in the entrance channel cause fluctuations in the reaction cross section. This can be dealt with by examining the ratio of fission to capture cross section, called $\alpha^{-1}$ in the literature. This analysis was attempted in Ref. [11] which found that large fluctuations still were present in the ratio. Another possibility is that the fluctuations are due to individual states in the second well acting like the resonant transition states in Eq. (2). Such resonance 
fluctuations are well known in fission under the barrier, but the common assumption is that the widths of these resonance become too large to play a role above the barrier. So far there is no theory to calculate these widths, but it is not out the question that theoretical tools can be developed to estimate these widths using phenomenological Hamiltonians based on nucleonnucleon interactions.

\section{Acknowledgments}

This material is based upon work supported by the US Department of Energy, Office of Science, Office of Nuclear Physics, under Contract No. DE-SC0012704 (BNL).

\section{References}

[1] See for example: O. Bouland, J.E. Lynn and P. Talou, Phys. Rev. C 88 054612 (2013).

[2] N. Bohr and J.A. Wheeler, Phys. Rev. 56426 (1939).

[3] D.A. Hill and J.A. Wheeler, Phys. Rev. 891102 (1953).

[4] T. Ericson, Ann. Phys. (NY) 33390 (1963).

[5] C.D.Bowman, G.S.Sidhu, M.L.Stelts, and J.C.Browne, 3rd Conf.Neutron Cross-Sections+Tech.,Knoxville 1971 Vol.2, p.584.

[6] R.B. Perez, et al., Nucl. Sci. Eng. 55203 (1974)

[7] M.S. Moore, et al., Phys. Rev. C 181328 (1978)

[8] Y. Alhassid, Rev. Mod. Phys. 72895 (2000).

[9] P. Fessenden, W. R. Gibbs, and R. B. Leachman, Phys. Rev. Lett. 15796 (1965).

[10] M.S. Moore, et al., Phys. Rev. C 30214 (1984).

[11] G.F. Bertsch and T. Kawano, arXiv:1701.00276 (2017). 